wards entirely recovered without having a trace of functional deficiency. The inference therefore to be drawn from this fact is that the morbid process did not occupy the cerebral lobes themselves, but was situated outside them. The train of symptoms, I think, are best explained on the supposition that the disease was a growth probably attached to the bone or membranes over the post-frontal and parietal regions of the right cerebral hemisphere. This, by general intra-cranial pressure, cansed the intellectual and sensory deficiencies, the universal debility, and the changes in the fundus of the eye. By special influence on the cortical motor centres it induced irritability of the grey substance without actual destruction, hence the paroxysms of convulsions in the parts deriving from them their motor activity. The same local pressure was also sufficient to cause permanent paresis of the same, which disappeared when the foreign body was subsequently absorbed, leaving the structures, of which it for a time suspended the functional activity, unimpaired.

The nature of the morbid growth must, from absence of pathological data, remain in doubt. Careful inquiry failed to elicit any trace of syphilis in the family history, but the appearances, progress, and termination of the case seem to point to that disease as constituting the local lesion of the brain. On the right forehead of the child there was a marked prominence of the bone, and the edges of the anterior fontanelle were raised and thickened in a manner highly suggestive of syphilitic infection. The termination and result of treatment might also by many be considered corroborative of this view. The patient throughout was treated chiefly with the bromide and iodide of potassium, and recovered under their influence, but whether as a result of the drugs or not I shall not attempt to determine. It is the habit of some authors to found a diagnosis upon the result of treatment, maintaining, for example, that in doubtful cases if a certain disease improve upon an anti-syphilitic remedy, that this in itself is evidence of the correctness of the suspected diagnosis. Such reasoning appears to me to be unscientific, and likely to lead to error, as it assumes as a fact what is by no means universally admitted.

Dr. Hughlings Jackson considers that the presence of double optic neuritis accompanied with unilateral convulsions is strong evidence in favour of specific disease. Doubtless it may be the fact that these are frequently associated, as syphilitic tumours are the most common cause of such local cerebral disorders; but from such symptoms we cannot diagnose syphilis to be present, but can only assert that gross lesion of some kind in the encephalon, involving the grey matter, is probable.

The general conclusions which seem permissible in this case are as follows: 1. That all the symptoms were due to cerebral disease. 2. That the lesion of the brain was gross, and not merely functional in character, and probably consisted of a morbid growth attached to the membranes or bones of the calvaria. 3. That this was of sufficient bulk to cause symptoms of general intra-cranial pressure. 4. That the lesion was localised, and probably limited to the cortical portion of the right post-frontal and parietal regions. 5. That the symptoms were induced by pressure on, but not destruction of, the nervous elements. 6. That recovery was due to absorption of the morbid product, and subsequent restoration of function of the cerebral convolutions. 7. That the disease was probably syphilitic in character.

\section{ON RUPTURE OF THE URINARY BLADDER}

BY JOHN A. MACDOUGALL, M.D. EDIN., CONSULTING SURGEON TO THE CUMBERLAND INFIRMARY.

IN his elaborate paper on Rupture of the Urinary Bladder Mr. Rivington, in THE LANCET of Nov. 4th, 1882, thus refers to the supposed cases of this injury which $\mathrm{I}$ published in the Edinburgh Medical Journal for January, 1877 :--" In neither of Dr. Macdougall's cases is there any satisfactory evidence of distension of the bladder at the time of the accident, and some of the most characteristic primary symptoms of rupture were conspicuously absent. Few surgeons would have any hesitation in setting aside the first case related. The second case rests mainly on the inability to micturate, the presence of blood in the urine, the small quantity of urine drawn off by the catheter, and the removal after an interval of a few hours of twenty-one ounces of fluid by means of the aspirator. Dr. Max Bartels carefully excludes the case from the category of intra-peritoneal ruptures, but seems willing to admit that it may have been an extra-peritoneal rupture; but even this view is attended with difficulties, to my mind insuperable. I believe that there was some unusual condition interfering with the withdrawal of the urine by the catheter, and that the aspirator entered the bladder itself." In reply I must first somewhat epitomise the cases, and having done so draw attention to the points which, in my opinion, still substantiate the original diagnosis.

CASE 1 was under the care of the late Dr. Thom of Brampton, and was published from bis own notes with the authority and acquiescence of Mr. Page, who saw the patient in consultation. "R. B-, a farmer, who had been drinking a good deal at market, left for home at 3 P.M., but stopped for a time at a public-house on the way. In passing through a gateway he was thrown from his cart, which was heavily loaded, and the wheel, he says, passed over his abdomen. He lay for about an hour, was then found, and carried home at 8 P.M. I saw him two hours afterwards. He was on his back in bed, still intoxicated, but complaining of great pain in the belly and of great desire to pass water, which, however, he had not been able to do. On being questioned, he stated that he had last made water at half-past two o'clock. There was a bruise extending from the left crista ilii across the pubes; a deep abrasion on the dorsum of the penis; and a fracture of the ilium, not extending into the true pelvis. There was no blood at the orifice of the urethra. A flexible catheter was introduced without a stylet, but was withdrawn, as it would not enter the bladder. It was easily introduced with a stylet, and was felt to pass along the prostate when examining per rectum. No urine flowed at first, but when pressure was made on the bladder six ounces came away, deeply coloured with blood. The catheter did not move freely in the bladder, and it required constant pressure to get the urine away." At 3 P.M. the following morning, within twelve hours of his injury, his state is thas reported : "Pulse 100, weak, distended abdomen; no bladder distension. Two ounces of urine were drawn off with the catheter, less bloody than the last." At 11 A.M. a still smaller quantity of urine was withdrawn. At half-past one on Thursday-that is, within twenty.four hours of the receipt of his injury-Mr. Page saw him in consultation, and his condition then was, "Pulse 140 , respiration 40, not entirely thoracic, but nearly so; abdomen very tender, much distended, and tympanitic, except for two inches above the pubes, where it was dull." The opinion was formed that the bladder was ruptured, and for the following reasons:-1st. The patient's bladder was probably much distended at the time of the accident, as he had been drinking freely, and had made no water for some hours. 2nd. The wheel of the cart had passed over the region of the bladder with sufficient force to fracture the ilium. 3rd. Only six ounces of urine came by the catheter when it was first used (seven hours and a half after he had last passed water), and only flowed when pressure was made above the pubes. 4th. There were signs of severe peritonitis.

CASE 2 was under my own observation in the Cumberland Infirmary, and may thus shortly be related :-J. B-C a waggon driver, aged twenty-three, was admitted into the Cumberland Infirmary on August 30th. Late in the afternoon of the day previous, while in charge of an empty waggon, one of the horses attached took fright, and striking him knocked him down. Two wheels passed over him, one over the lower part of his abdomen, the other over his right arm. After an interval of fully three hours from the receipt of his accident he was seized with pain in the belly. With this came urgent and intense desire to make water, but all attempts to do it failed. Many miles romoved from medical assistance, and feeling sick and weary, he retired to rest, and, strangely enough, he slept fairly well during the night. With morning, however, came an aggravation of all his symptoms, and he sought the advice of Dr. Robertson, of Penrith. He, learning from him his total inability to pass urine, used a catheter, but only succeeded in removing four ounces. This was quite twenty hours after the last time he had made water, and what he withdrew was deeply tinged with blood. J. B- entered the ward while I was making the morning visit, and so freely and apparently easily did he walk that I never suspected his condition was so serious. The report of his state as then observed is copied from the ward journal :- "The patient is a strong healthy-looking man, well nourished and well developed. 
His integument is warm and dry; his temperature is $99^{\circ}$. He has an anxious pinched expression of countenance, and marked nervous $t$ witchings of the muscles of the face...... Examination of the abdomen shows percussion dulness absolute in the right iliac region, the hypogastric region, part of the umbilical region, and to a less marked degree in the left iliac region; the slightest touch gives him pain, pressure he cannot bear. This tenderness is most marked towards the right iliac fossa. A full-sized catheter with a short curve was carefully introduced, and about one ounce of bloody urine withdrawn." The history and the examination left little doubt that he had rupture of his bladder. That the matter might be rendered, if possible, more certain, he was put under chloroform, and passing my left hand into the rectum well beyond the prostate with a short beaked catheter in the bladder, the empty and contracted condition of the viscus was readily demonstrated. In order to remove the urine effused into the peritoneal eavity, a No. 2 aspirator needle was introduced in the centre line an inch above the pubes. Bloody fluid escaped slowly through it, and when nearly five ounces had been withdrawn the instrument, partly due to the consistence of the fluid, partly to some fault in the suction apparatus, struck work. This was matter for regret, as the condition of the patient was such that the removal of the fluid was imperatively demanded. Chemical examination proved the fluid removed to be urine highly charged with blood. I visited him again some hours later, and found him markedly worse. Vomiting, which had occurred for the first time shortly after I had left him, had rendered the pain more severe and the vital depression was greater. Two modes of procedure now suggested themselves: either to use the largest sized trocar of the aspirator so as to give ready vent to the accumulated fluid, or having freely opened the peritoneal cavity to sponge it thoroughly out and make an attempt to reach and close the wound in the bladder. Mr. Page, who now saw the case with me, agreed that, failing a satisfactory result from the first procedure, we should adopt the second. The trocar was accordingly introduced obliquely downwards and backwards close above the pubes, the aspirator affixed, and by it twenty-one ounces of fluid were withdrawn. All abdominal dulness had now disappeared, and the acute tenderness seemed less. A soft catheter was passed and retained. - August 3lst Pulse 120, temperature $1002^{\circ}$. The urine, which still contains much blood, passes freely by the catheter. The tenderness of the abdomen on pressure is still extreme, but there is now no dulness. Evening: The pulse and temperature had risen, and it was ascertained that no urine had passed by the catheter for some hours. With the idea that the instrument had become blocked it was withdrawn, washed, and introduced, but with no better result. Percussion revealed a little return of the dulness above the pubes, and fearing that the urine was again finding its way into the peritoneum, the house-surgeon, Mr. Spence, used the aspirator, and withdrew several ounces of sanguinous fluid. The removal of this, which examination proved to be largely sero-purulent, was followed by benefit, for in a short time the urine again passed guttatim by the catheter. From this time the urine became daily less bloody until on September 6 th, having himself withdrawn the catheter, he passed nearly a pint of clear urine. He had, however, a terrible illness, intense peritonitis, complicating acute septicæmia, and for many days his condition seemed a very hopeless one. Ultimately his recovery was perfected, and on Sept. 22ad it is noted :- "The temperature is seldorn over $101^{\circ}$, and his general condition is better. Over the lower part of the abdomen there is still perceptible dulness on percussion, and the doughy feeling on palpation is very evident-both signs, I imagine, of peritoneal matting and thickening."

The points in which Mr. Rivington finds these cases defective are the following. First, no satisfactory evidence of distension of the bladder at the time of the accident, and, second, that some of the most characteristic primary symptoms of rupture were conspicuously absent. As regards the first objection, I can only say that in Dr. Thom's case it does not hold good. In the relation of his own cases, and in referring to those of others, Mr. Rivington dwells constantly upon the intimate connexion between ruptare of the blidder and drinking, and here we have present this very association. So notable was this, that the primury reason Dr. Thom gives for considering the case as one of rupture is "that the patient's bladder was probably much distended at the time of the accident, as he had been drinking freely and had made no water for some hours."

In the case of J. B- we did ascertain, although the fact is not mentioned in the published notes, that he had not made water for some hours before the receipt of his in. jury. That the presence of distension is, however, by no means necessary to the production of this lesion many cases prove, while those of Mr. Cusack and Mr. Heath go far to establish the fact that it may occur with an all but empty bladder. With reference to the second objection, "that some of the most characteristic primary symptoms of rupture were conspicuously absent," I can best reply by stating that after a careful perusal of Mr. Rivington's own cases where the diagnosis was verified by post-mortem examination, I find that with the exception of the sensation of rupture having occurred, no characteristic primary symptom which he records is absent in my own. Both patients had been subjected to crushing violence in the region of the bladder; both had, the one earlier, the other later, severe pain in the abdomen, with urgent but futile desire to pass water; and in both the urine, when withdrawn, was not only most notably small in quantity, but it was deeply tinged with blood. Peritonitis, severe in its character, was the sequel; and though recovery followed, rare as we all know such an event to be, still it is by no means an unknown one.

Of Dr. Thom's case I can say little more, as I never saw the patient; but of the accuracy of the diagnosis in J. B-'s case I have no doubt. Dr. Robertson, who used the catheter twenty hours after the man had last made water, withdrew only four ounces of bloody urine. Three hours after I could only get one ounce, and yet there was no difficulty in the introduction of the instrument, although there was when an attempt was made to depress or rotate it.

Mr. Rivington's idea is that I aspirated the bladder. The following are my reasons for believing that I did not:-The abdominal dulness was not that of a distended bladder; it was scarcely marked in the umbilical region at all, while it was absolute in the right iliac region, and it was not absent in the left. A careful examination with a hand in the rectum and a catheter in the bladder demonstrated the viscus empty, and that there was entire absence of fluctua. tion or bulging in the region above the prostate, a condition which must have existed had the bladder contained what the aspirator withdrew, nearly thirty ounces of fluid. When the aspirator was used, a No. 2 needle was chosen-a size which in my experience gives ready exit to normal urine-but in this instance so thick was the fluid that it would not flow through it. It was only when the largest sized trocar was introduced that free evacua. tion took place. A healthy and continent bladder containing bloody urine, with a density so great as it was in this case, must be rare indeed. Added to all this is the further evidence afforded by the procedure of $M r$. Spence. Twenty-four hours after the primary tapping he again aspirated the abdomen. That he, too, could have pierced the bladder is impossible, inasmuch as the urine had up to the short period of a few hours been flồwing freely through the retained catheter, and yet what he withdrew was a urinous fluid largely sero-purulent. There was, to my mind, in this not only a demonstration of the existence of urine in the peritoneal cavity, but of acute peritonitis as a consequence. At no period in the whole progress of the case was there any evidence of urinary infiltration, which there must have been had the rent been extra-peritoneal and I therefore discard as quite untenable the opinion of Dr. Max Bartels.

Thus far, but before closing, I would venture to call Mr. Rivington's attention to the record of his own case of re. covery after extra-peritoneal rupture. The story is this: $-A$ woman sustains a fall while pregnant, and three weeks after she has a premature confinement. Parturition is favourable, but she is ill directly afterwards and suffers from a constant pain in her side. Suddenly the pain removes to the right groin and abscesses form, having discharging sinnses. One day urine was found issuing from these sinuses. Ultimately she left the hospital having made a complete recovery.

Surely this case can hardly be reckoned as one of ruptured bladder. The causes of rupture are violence or over-dis. tension. This woman has no history of laving been subjected to the former, ánd under constant observation in a hospital she could hardly have suffered from the latter. Moreovel. vad over-distension been the cause the chances are that the bladder would have given way above the line of 
the peritoneal reflection. The case reads as one of a bladder perforated by the necrotic process in the walls of a puerperal peri- or para-metric abscess, the urine finding issue by the already established sinuses. Such cases are not un. known in gynæcological practice.

Carlisle.

\section{FOREIGN BODIES IN THE AIR-PASSAGES,} WITH ESPECIAL REFERENCE TO CASES OF "PUFF AND DART."

\section{BY ROBERT BRUCE, M.D., F.R.C.S.E.}

EXAMPLES of foreign bodies becoming accidentally lodged in the air-passages are of common occurrence, but cases where the offending body is a dart have not been so frequently met with. It would appear, however, from some instances which have been recorded lately, that it is not so nnlikely to happen as one might imagine; and, from the danger which attends an accident of this kind, it would seem desirable to give greater prominence to it than has hitherto been done.

CASE 1.- On Nov, 29th, 1879, a boy eleven years of age was amusing himself with a favourite toy called "puff and dart." when, most unfortunately, he happened to take a full inspiration while his mouth was applied to a tube through which he was about to blow a dart. $\mathrm{He}$ immediately exclaimed that he had swallowed it. Violent fits of coughing at once ensued, and he instinctively made vigorous efforts to retch, hoping by this means to bring the weapon up again. When seen by me on the following day, for the first time, he appeared so easy that I felt inclined to share the little patient's belief that the dart (composed of a strong needle $1 \frac{3}{8}$ in. long, with a body of worsted firmly fastened to its blunt end) had really gone into his stomach. From the absence of any difficulty in breathing or fit of suffocative cough, and as the voice was unaltered, being neither hoarse nor croupy, it was evident that both the larynx and trachea were free. Auscultation, however, disclosed that air entered very imperfectly into the left lung as compared with the right. It then became clear that the needle with its appendages had undoubtedly found its way into the left bronchus, and the case at once assumed the gravest aspect. On the one hand, there was the danger of suffocation at any moment should the foreign body be ejected against the rima glottidis; while on the other, were it to remain for any length of time in the lung the most serious consequences were to be apprehended. In consultation with the late Professors Spence and Sanders, the diagnosis was confirmed, and it was agreed that the trachea ought to be opened without delay in order to guard against the first of the dangers already mentioned, as well as to afford a readier outlet for the needle should it by any chance be expelled from its resting-place, and at the same time to permit of careful probing in the hope of ascertaining its position, and possibly dislodging it. In accordance with this view of the case, tracheotomy was performed by Professor Spence, assisted by Dr. John Duncan, on Dec. 4th, under chloroform. The thermal cautery was employed for this purpose, and proved to be a very efficient agent, its action being almost bloodless, which was of great importance, as our patient was by no means of a robust constitution and had a decidedly anæmic appearance. Immediately after opening the trachea, a probe was passed cautiously downwards in search of the needle, but its position could not be discovered, and there was no suitable instrument available at the time for attempting to lay hold of it, while the extreme irritability of the parts on the introduction of any instrument, as evidenced by the supervention of violent fits of coughing, and ejection of quantities of frothy mucus, gave warning that prolonged operative interference would not be tolerated. Great prostration followed the combined effects of the operation and the chloroform, from which the patient but slowly rallied. The constitutional disturbance which ensued was correspondingly severe, and was evidenced by increase of temperature $\left(102^{\circ}\right)$, rapidity of pulse (150), and quickened respiration. The pulse fluctuated somewhat, but did not approach its normal frequency until three weeks after the operation ; sickness and retching in addition were of frequent occurrence and occasionally attacks of diarrhoea supervened. On Jan. 10th, 1880, small quantities of blood were expectorated. The fits of coughing, attended with expectoration of a large amount of muco-purulent matter, went on continuously, but the general condition improved so far that by March 22nd it was considered that another attempt to remove the foreign body might be justifiably made. On this occasion various kinds of forceps were employed, but none of them appeared to reach far enough down to be attended with any measure of success, and so had to be discontinued. In May our patient went to the country and recruited considerably in health. On his return it was decided that another chance should be afforded him of obtaining permanent relief, there having been no symptoms indicating any appearance of loosening and spontaneous expulsion, which had been so long looked for. Therefore on June 2nd Professor Spence and Dr. Duncan again met to resume operative measures, and under chloroform careful and repeated explorations were made to ascertain the position of, and if possible to remove, the needle. At this time some specially constructed instruments, according to my directions (as depicted in the accompany. ing woodcut), were made use of. These were two in

Frg. 1.

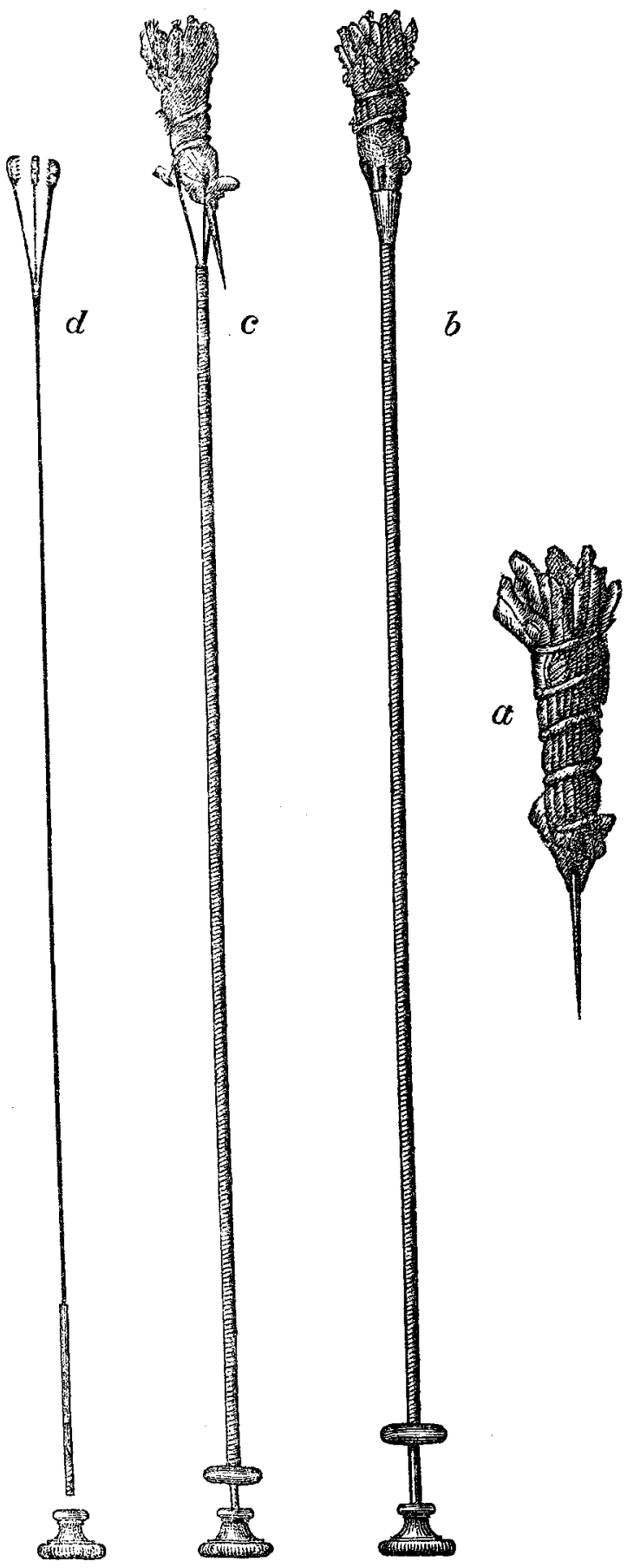

number, and consisted each of a steel wire spiral spring, twelve inches in length, forming a flexible tube (c), through which was passed a steel wire with three prongs (d) at its terminal point, which could be expanded or closed at will by raising or depressing the button handle. One of the spiral tubes $(b)$ was made bell-shaped at the end, so that in passing downwards, should it fortunately cover the 